\title{
Pharma Industrial Internet: A Reference Model Based on 5G Public Private Partnership Infrastructure, Industrial Internet Consortium Reference Architecture and Pharma Industry Standards
}

\author{
Pasi Kemppainen
}

NNE Pharmaplan, Copenhagen, Denmark

E-mail:pske@nnepharmaplan.com

Received 22 February 2016;

Accepted 21 March 2016

\begin{abstract}
Pharma industry is undergoing a major transformation called 'The new pharma reality' caused by the changes in global pharmaceutical business ecosystem and strive for the improved regulatory compliance and competitiveness. The current manufacturing and enterprise IT systems have not been satisfactorily able to deliver on the new requirements and this is leading pharma companies investigating the benefits of the current trends of digitalization, IoT and enhanced networking capabilities. This paper introduces the concept of Pharma Industrial Internet, a reference model for applying the 5G PPP Infrastructure, Industrial Internet Consortium Reference Architecture and key pharma industry manufacturing standards for specifying, modeling and building pharma industry specific industrial internet solutions. By applying the reference model, pharmaceutical manufacturers will be able to improve their competitiveness while meeting the global regulatory and manufacturing quality requirements. The paper provides a holistic view on the digitalization transformation taking place in the pharmaceutical industry, the technical enablers driving the transformation and the new service infrastructures and
\end{abstract}

Journal of NBICT, Vol. 1, 141-162.

doi: 10.13052/NBICT.2016.008

(C) 2016 River Publishers. All rights reserved. 
value-add enterprise services helping the pharma companies to compete in the new pharma reality.

Keywords: Industrial internet, the new pharma reality, pharma manufacturing IT, 5G, 5G PPP, Industrial Internet Concortium, OPC UA, ISA, GS1.

\section{Introduction}

Pharma business and manufacturing are undergoing major transformation on multiple concurrent levels. The era of conventional blockbuster medicines is ending and the big pharma focus is shifting towards high value but low volume biomedicines. At the same time, the increasing price pressure caused by the tightening market price regulations and patent expiries is forcing small and mid-size pharma companies to increase their number of products to maintain the revenue and profitability level. In addition, the diverse and fast changing global regulations in pharma manufacturing and supply chain compliances are demanding infrastructure changes beyond the current readiness and capabilities. In the pharma industry this concurrent transformation is called the new pharma reality.

Pharma manufacturing is understandably very regulatory, industry standards and standard operation procedures driven due to the stringent quality requirements and patient safety. However, the current pharma IT infrastructures, which can still date even decades back, are not any more sufficient for meeting the new pharma reality requirements. The pharma manufacturing IT/OT (Information Technology/Operations Technology) infrastructure implementations are already highly complex with increasing number of connected equipment, and internal and external system integrations. The pharma ecosystem is also highly networked with the pharma industry specific requirements e.g. for the collaborative data management and regulatory compliance reporting. Therefore, any pharma architecture framework to be successfully adopted within the industry needs to be based on the global standards, proven common architectures and open technologies.

While the pharma industry is undergoing major transformation, the emerging networking technologies and their utilization is changing the industries and consumer behavior. Especially, the emerging $5 \mathrm{G}$ standardization promises to create a globally standardized networking infrastructure for the improved industrial internet and Internet of Things (IoT) services. These capabilities are the foundation for implementing end-to-end digitalized pharma solutions. 
The purpose of this paper is to provide a holistic view on the digitalization transformation taking place in the pharmaceutical industry, the technical enablers driving the transformation and the new service infrastructures and value-add enterprise services helping the pharma companies to compete in the new pharma reality. The paper introduces how 5G PPP Infrastructure, Industrial Internet Consortium Reference Architecture and key pharma industry manufacturing standards, such as ISA, OPC UA and GS1 standards, can be used for specifying, modeling and building pharma industry specific industrial internet solutions. Pharma industrial internet reference model is a holistic and technology agnostic model covering the high-level capabilities and architectures from the pharma manufacturing IT to the enterprise IT and business integration between the stakeholders in the pharma ecosystem. The goal with the Pharma Industrial Internet reference model is to help pharma companies to conceptualize and implement pharma regulatory compliant industrial internet solutions using global industry standards and proven architecture frameworks fulfilling the future IT/OT infrastructure, business operations and expenditure requirements for the new pharma reality and beyond.

The rest of this paper is structured as follows. Following the motivation part in the introduction, the new pharma reality is discussed in Chapter 2. The new pharma reality sets the requirements for the future solutions like the Pharma Industrial Internet. Chapter 3 presents the complexity of the current pharma supply networks to illustrate the challenges when implementing Pharma Industrial Internet solutions. Chapter 4 then presents Pharma Industrial Internet with the key characteristics and technology enablers, describes how 5G, IIRA and pharma industry standards contribute to realize Pharma Industrial Internet. The chapter also specifies Pharma Industrial Internet Reference Model using IIRA model, ISA manufacturing automation industry standards, and GS1 and OPC specifications. Chapter 5 describes different Pharma Industrial Internet ecosystem and services based on the medicinal product life-cycle from R\&D to patients. Finally, chapter 6 concludes describing the implementation aspects for Pharma Industrial Internet linking the new pharma reality requirements with the technology and infrastructure enablers, and respective business drivers and capabilities.

\section{The New Pharma Reality}

The recent study by NNE Pharmaplan [1] investigated global pharma companies' perspectives of the future successful pharma manufacturing sites. 
The study identified changing expectations of success moving from site stability to site agility with three key requirements:

1. 'Flexible production', a site's ability to accommodate changes in production demands,

2. 'Integrated quality', balanced and integrated quality systems; and

3. 'Entering new domains', having the ability to quickly absorb knowledge to implement new practices.

Pharmaceutical companies are clearly identifying that due to the external and internal drivers (see Figure 1), their competitiveness is at stake. This is partly due to the fact that the current pharma product supply systems consisting of manufacturing, finished goods packaging, warehousing, logistics and supporting back-end pharma IT infrastructures, are not capable in supporting the changing expectations.

The external drivers concern the ecosystem, regulatory and market specific changes where the pharma companies have very little influence. Especially important are the increased regulatory pressure and increased competition from generics. Pharma companies need to comply with the regulatory requirements to be authorized selling their medicines, and there's no middle ground: the companies are either compliant or not. Therefore, the increased and hardening regulatory requirements can be a risk for the business continuation. The internal drivers can be mostly managed by the pharma manufacturer. These include strive for the cost-efficiency and strive for quest for key differentiators from the competition. However, there are already huge investments in place for the pharma manufacturing and enterprise IT which are not able to deliver the cost-efficiency and capabilities expected for the future competitiveness.

Additional substantial problem is that the current manufacturing infrastructures have been validated and regulatory audited to produce the medicines. Therefore, replacing the current infrastructures and solutions is a huge business risk. This has led to a dilemma where the manufacturers are in-between trying
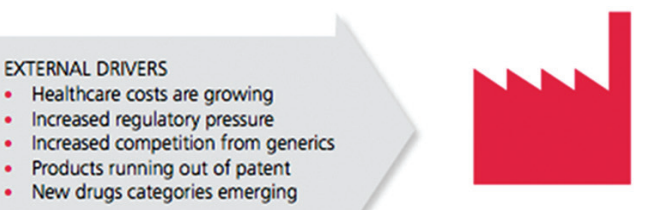

- Global production

- Standardisation

- Quest for key differentiators

Figure 1 External and internal drivers for pharma industrial internet [2]. 
to stretch the current infrastructures as much as possible but still need to invest in the new capabilities to be competitive in the future.

This is called as 'the new pharma reality' and it can't be accomplished without completely rethinking the pharma product supply delivery infrastructure and business operations. This will lead to Pharma Industrial Internet infrastructures and solutions.

\section{Pharma Product Supply Network}

The best way to illustrate the new pharma reality and digitalization transformation in the pharma industry is to describe it from the product lifecycle and supply network point of view. This also helps to understand the differences between industrial internet and Internet of Things (IoT). Industrial internet describes the digitalization of the enterprise IT/OT infrastructures (from manufacturing the "things" to supplying the "things" to the market), whereas IoT describes the digitalization of the end-user pharma related "things" and processes using IT services and smart connected devices, such as in drug development, clinical trial, patient care and wellbeing services. This paper focuses on describing how the industrial internet transforms the pharma manufacturing and supply network.

Global pharma business today is already very complicated in terms of ecosystems and supporting product supply infrastructures. In the Figure 2 it

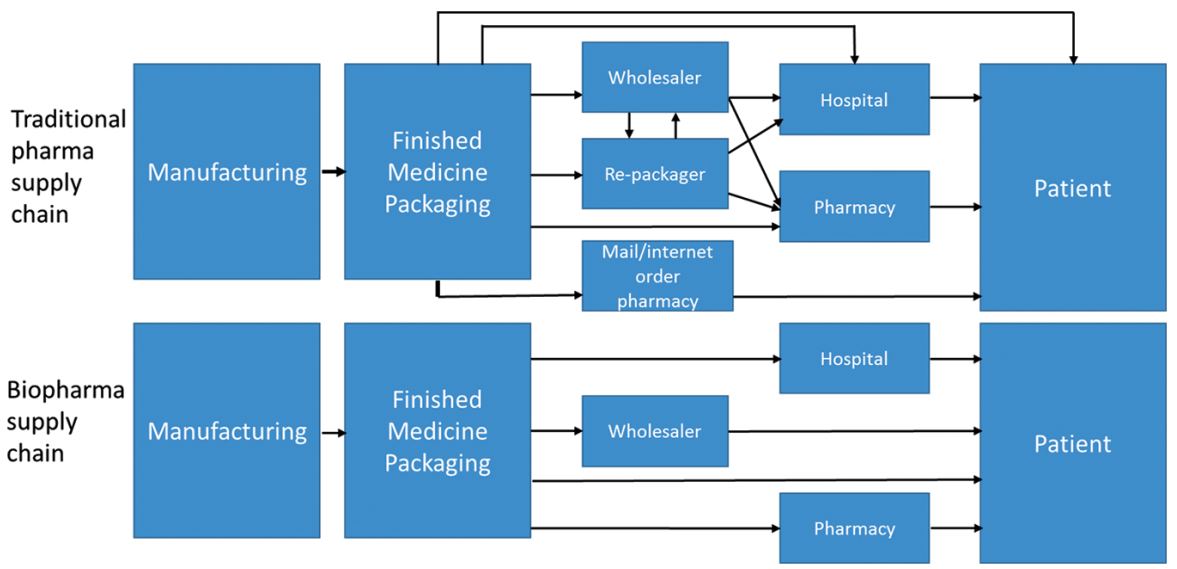

Figure 2 Examples of the current pharma product supply chains in traditional pharma and biopharma businesses. 
is illustrated a simplified current product supply network that takes care of delivering the medicinal products from research and development to patients. It constitutes of the following key operations and supporting infrastructures:

- Primary manufacturing for producing the bulk medicine.

- Finished medicines (goods) manufacturing, also called as packaging.

- Finished medicines supply chain, consisting of warehousing and logistics.

- Supporting back-end IT systems such as ERP, PLM, partner collaboration, business intelligence and regulatory reporting systems.

The high infrastructure complexity is multiplied due to the extensive number of global collaboration partners. There exist a large variety of different business models in terms of contract manufacturing, packaging, third-party warehousing and logistics, and sales channels. In addition, with the increasing introduction of advanced healthcare related services using the Internet of Things (IoT) capabilities, it can be envisioned that the complexity of managing the whole pharma product lifecycle and supply will even increase.

The new pharma manufacturing and product supply service infrastructures and ecosystems transforming the current pharma product supply are presented in Chapter 5.

\section{Pharma Industrial Internet}

Pharma Industrial Internet is a major industry transformation which encompasses many concurrent technological, ecosystem and service model changes [3]. This chapter describes changes specifying first the key technology enablers and solution characteristics and then the mapping between industrial internet reference architecture and pharma standards regarding the existing pharma enterprise solutions. Chapter 5 explains in more detail the implications in the current pharma ecosystem and service models.

\subsection{Key Technology Capabilities, Enablers and Solution Characteristics in Pharma Industrial Internet Solutions}

Industrial Internet can be envisioned as the convergence of three technological capabilities: sensor-driven computing, industrial analytics and intelligent machine applications [4]. In manufacturing intensive industry this means integrating complex physical machinery such as robots with networked sensors and software/cloud services enabling innovative business solutions in manufacturing, enterprise IT, analytics and value chain management. 


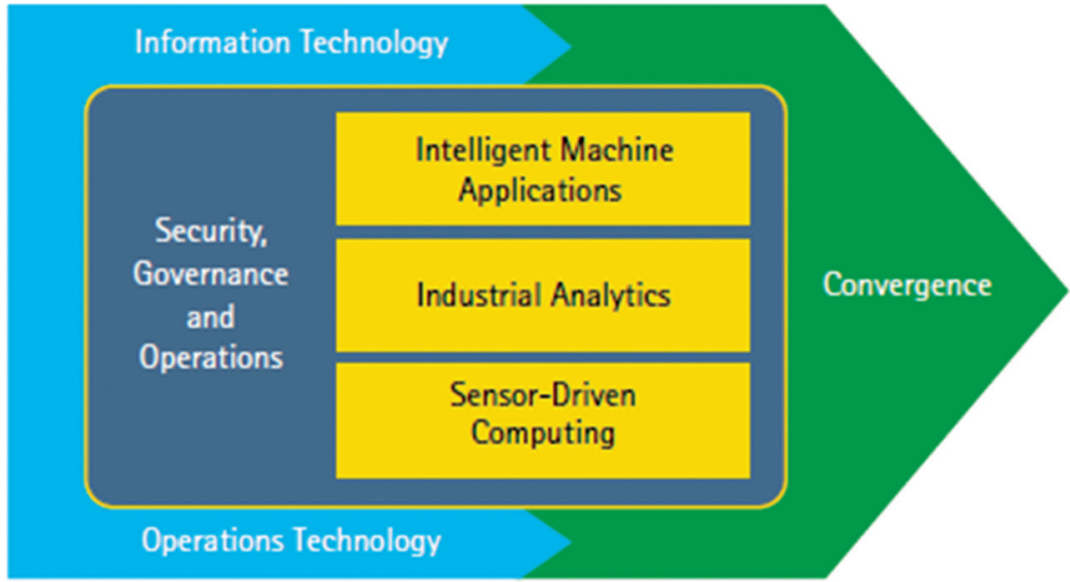

Figure 3 The key industrial internet technological capabilities [4].

The key pharma industrial internet technology enablers and platforms can be categorized as [4]:

1. Information Technology, such as

- Existing enterprise IT systems (ERP, CRM, supply chain planning and management, business analytics, etc.)

- Manufacturing IT management and integration (MES, MII, OEE, etc.)

- Big data and cloud solutions

- Mobile technologies

2. Operations Technology, such as

- Distributed Control Systems

- SCADA sensors and equipment

- Advanced machinery/robots for full automation

3. Security, Governance and Operations, supporting the key industrial internet enablers such as

- Intelligent machine applications

- Industrial analytics

- Sensor driven computing

Based on the key capabilities described above, the key solution characteristics based on the technology enablers and platforms for the Pharma Industrial Internet solutions can be then described as 
- Machinery intelligence with independent application logic and data management at the manufacturing hardware level ("edge/fog computing"),

- Manufacturing intelligence facilitated by the analytics for improved transparency in manufacturing processes,

- Real-time data with the fast and low-latency communications with the back-end manufacturing and enterprise services,

- Flexible service distribution utilizing public and private cloud platforms for the most efficient and scalable service delivery,

- Connectivity with seamless integration with the manufacturing/enterprise services, business partners and end-users/devices,

- Inherent security in all levels of communications and services transactions, and

- Future proof being based on the pharma industry standards and specifications to ensure the systems and services integration and semantic interoperability.

There is no single architecture or framework available which can fulfill the all key characteristics alone. Hence, the use of complementing architectures and frameworks is needed. The Pharma Industrial Internet reference model uses three different architectures for specification and modeling (see Figure 4):

- 5G Public Private Partnership (5G PPP) [5] for the industrial internet ecosystem, network access and service delivery infrastructure,

- Industrial Internet Consortium Reference Architecture (IIRA) [6] for modeling the industrial internet services functionality and distribution, and

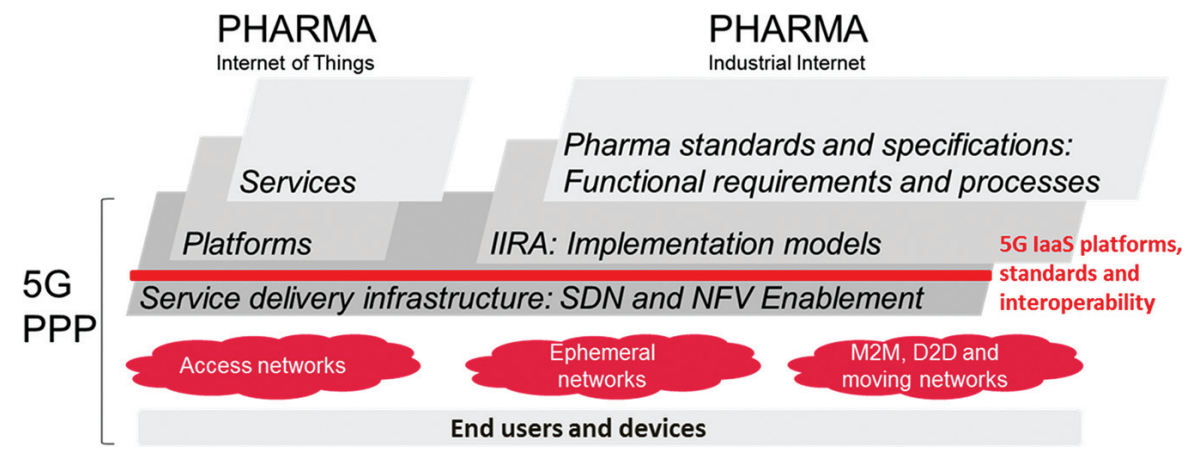

Figure 4 Pharma internet of things and industrial internet service infrastructures in 5G PPP ecosystem. 
- Standards and specification used at the pharma industry [7-10] to specify the functional requirements and processes for the implementations.

The rationale using 5G PPP for the underlying network and service delivery infrastructure is to have a global and standards based ecosystem for service provider agnostic industrial internet services. IIRA, on the other hand, specifies the key common viewpoints and functionalities for the industrial internet services which help in specifying and modeling the actual implementations. Standards and specifications used in the pharma industry already today are naturally the foundation for specifying the implementation user and functional requirements, and operational processes.

\subsection{Mapping the Characterics of 5G PPP, IIRA and Pharma Industry Standards with Pharma Industrial Internet Reference Model}

\subsubsection{G PPP}

The emerging $5 \mathrm{G}$ will bring new unique network and service capabilities. Firstly, it will ensure user experience continuity in challenging situations such as high mobility, very dense or sparsely populated areas, and journeys covered by heterogeneous technologies. In addition, $5 \mathrm{G}$ will be a key enabler for the Internet of Things by providing a platform to connect a massive number of sensors, rendering devices and actuators with stringent energy and transmission constraints. Furthermore, mission critical services requiring very high reliability, global coverage and/or very low latency, which are up to now handled by specific networks, typically public safety, will become natively supported by the 5G infrastructure. [5] 5G will integrate networking, computing and storage resources into one programmable and unified infrastructure. This unification will allow for an optimized and more dynamic usage of all distributed resources, and the convergence of fixed, mobile and broadcast services [5].

5G helps to specify the overall industral internet ecosystem and infrastructures with different means of accessing and utilizing the services. It also specifies key infrastructure enablers such as Service Defined Networking (SDN) and Virtualized Network Functions (VNF) for scalable and flexible (elastic) service infrastructure enablement and service provisioning. It can be anticipated that in addition to the seamless and pervasive networking services, the 5G service delivery infrastructure will provide standardized set of IaaS

(Infrastructure as a Service) capabilities and interfaces for implementing the 
IIRA industrial internet services. However, the specification of $5 \mathrm{G}$ service delivery infrastructure still in early stage and the final requirements and standardization remains to be seen.

\subsubsection{IIRA}

The Industrial Internet Reference Architecture (IIRA) is a standard-based open architecture for industrial internet services (Figure 5). To maximize its value, the IIRA has broad industry applicability to drive interoperability, to map applicable technologies, and to guide technology and standard development. The description and representation of the architecture are generic and at a high level of abstraction to support the requisite broad industry applicability [13].

The members of the Industrial Internet Consortium have defined an architecture framework that describes the conventions, principles and practices, based on ISO/IEC/IEEE 42010:2011 that facilitates evaluation, and systematic and effective resolution of stakeholder concerns, and guides communication about the IIRA [14].

There are four main categories:

- The business viewpoint covers concerns related to the business (chiefly requirements).

- The usage viewpoint covers how the system is used by operators [users].

- The functional viewpoint outlines the main functional blocks (about which, more below).

- The implementation viewpoint covers, unsurprisingly, implementation [13].

The functional viewpoint contains the functional blocks that make up the system. An organization developing an Industrial Internet system may not use all the blocks, but should at least consider whether the functional blocks

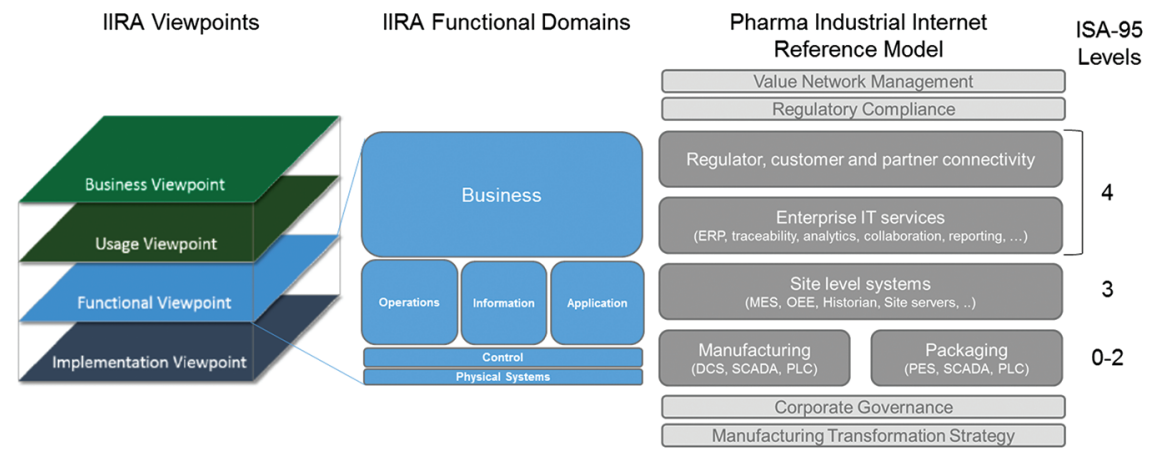

Figure 5 IIRA, pharma industrial internet reference model and ISA-95 compatibility. 
apply in their system. [12] The functional viewpoint is essential in enabling the mapping the IIRA functional blocks with the standards and specifications used in the pharma industry.

As depicted in the Figure 5, IIRA functional viewpoint is divided into five functional domains [14]:

- Control domain, representing the collection of functions that are performed by industrial control systems. The core of these functions comprises fine-grained closed-loops, reading data from sensors ("sense" in the figure), applying rules and logic, and exercising control over the physical system through actuators ("actuation"),

- Operations domain, representing the collection of functions responsible for the provisioning, management, monitoring and optimization of the systems in the control domain,

- Information domain, representing the collection of functions for gathering data from various domains, most significantly from the control domain, and transforming, persisting, and modeling or analyzing those data to acquire high-level intelligence about the overall system,

- Application domain, representing the collection of functions implementing application logic that realizes specific business functionalities, and

- Business domain, enabling end-to-end operations of the Industrial Internet Systems by integrating them with traditional or new types of Industrial Internet specific business functions including those supporting business processes and procedural activities.

These domains represent the foundation for the compatibility with the pharma standards and specifications.

\subsubsection{Pharma manufacturing and enterprise IT standards and specifications}

Pharma manufacturing and enterprise IT standards and specifications are obviously the foundation for implemeting the Pharma Industrial Internet solutions utilizing $5 \mathrm{G}$ networking infrastructures and IIRA implementation guidelines. In addition, there are naturally a lot of vendor and enterprise specific specifications due to the proprietary manufacturing and business processes. The positive side in the pharma implementations is that, being in compliance with the regulatory requirements, they need to be properly documented and validated. This is why the Pharma Industrial Internet reference model can be amended with enterprise specific requirements and architecures, including the legacy systems interoperability. 
The key manufacturing standards and specifications supporting the Pharma Industrial Internet are (Figure 6):

- ISA-95 [7], specifying the overall enterprise architecure for enterprise and manufacturing interoperability (standardizing the IT functionalities in levels 0-4 in Figure 6).

- ISA-88 [8], specifying the manufacturing batch control and recipe management (ISA-95 levels 0-3).

- ISA-99 [9], specifying the infrastructure security requirements and modelling (ISA-95 levels 2-3).

- GS1 EPCIS [10], specifying the enterprise data exchange interoperability internally and with the external business partners and regulators.

- OPC-UA [11], specifying the manufacturing hardware interoperability between manufacturing control systems and devices.

IIRA functional viewpoint and respective functional domains are the essential linkage between the IIRA guideline and pharma infrastructure modelling. Mapping the IIRA functional viewpoint functional domains with ISA-95 levels provides the Pharma Industrial Internet reference model a coherent linkage between the legacy the future infrastructure implementations enabling the migration to the Pharma Industrial Internet services and interoperability with the legacy implementations (Figure 6).

\subsubsection{Corporate transformation strategy and ecosystem management}

In addition to the technology and business requirements and viewpoints, it is important to emphasize that any successful infrastructure implementation

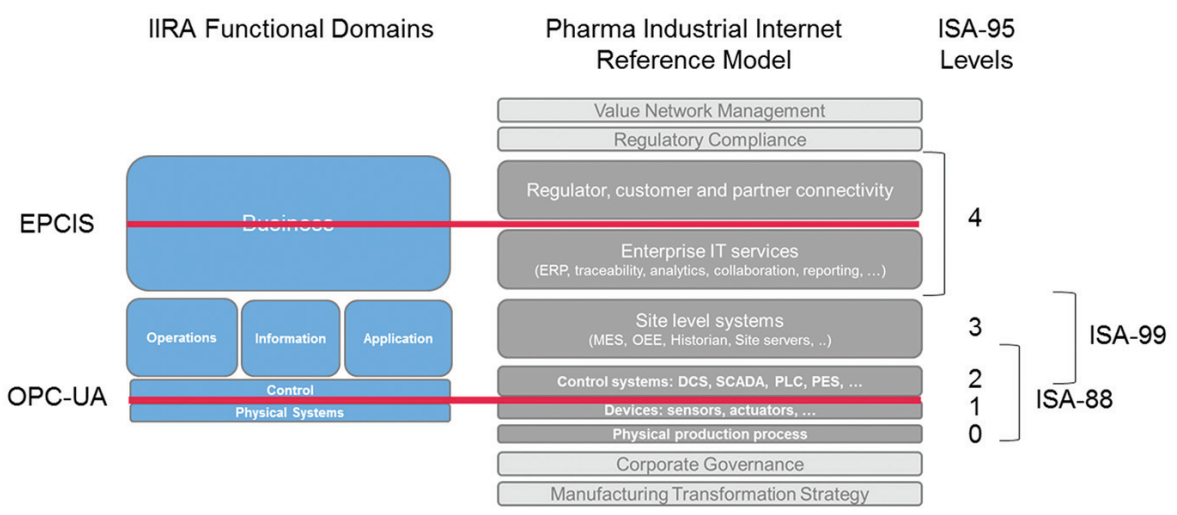

Figure 6 Mapping the key standards and specifications in pharma industry with IIRA and pharma industrial internet reference model. 
requires solid corporate strategy for the digital transformation ("digitalization") and, especially in the case of industrial internet infrastructures, the ecosystem management.

The industrial internet corporate tranformation strategy is essential is setting the business priorities and performance indicators for the actual implementation and operations. After the tranformastion strategy is formalized and ready to be operationalized, the following key capability is the corporate governance: getting the strategy implemented and corporate units accountable for the successful implementation (Figure 6).

Ecosystem management encompasses the engagement of the value network partners including suppliers, partners, regulators, end-users/patients and even competitors. Because the industrial internet infrastructures and solutions are highly networked, the external services interoperability within the value network is essential for the successful infrastructure implementation. Especially in the pharma industry, the regulatory compliance is the fundamental pre-requisite for the business continuation (Figure 6).

\section{Pharma Industrial Internet Ecosystem and Services}

Implementing the Pharma Industrial Internet is a long journey. It means not only implementing new service infrastructures and solutions, but there are numerous existing legacy solutions which obviously needs to be supported with the digitalization. And additionally, there is already another concurrent transformation taking place in healthcare, the Pharma Internet of Things. The outcome of these complementing digital transformations will be using the advanced technologies and services for creating better treatment and therapy possibilities in pharma $\mathrm{R} \& \mathrm{D}$ (Treatment 2.0), and providing the patients and healthcare professionals better products potentially combining advanced sensoring hardware with mobile and cloud services (Product 2.0).

To illustrate the ecosystem and different services therein enabled by the Pharma Industrial Internet, the product lifecycle model can be used as a baseline: how the industrial internet services can support getting the medicinal products from $R \& D$ to the end-users/patients, and supporting the product usage until the end of the product life cycle (Figure 7). The life-cycle model consist of two pharma ecosystems: Pharma Internet of Things and Pharma Industrial Internet. Pharma IoT consists of two complementing subecosystems namely R\&D and patient and end-user services. They both utilize the IoT technologies and ecosystems for improved services and products, and naturally for new business models. However, although Pharma IoT inherently complements Pharma Industrial Internet, it is out of the scope of this paper. 


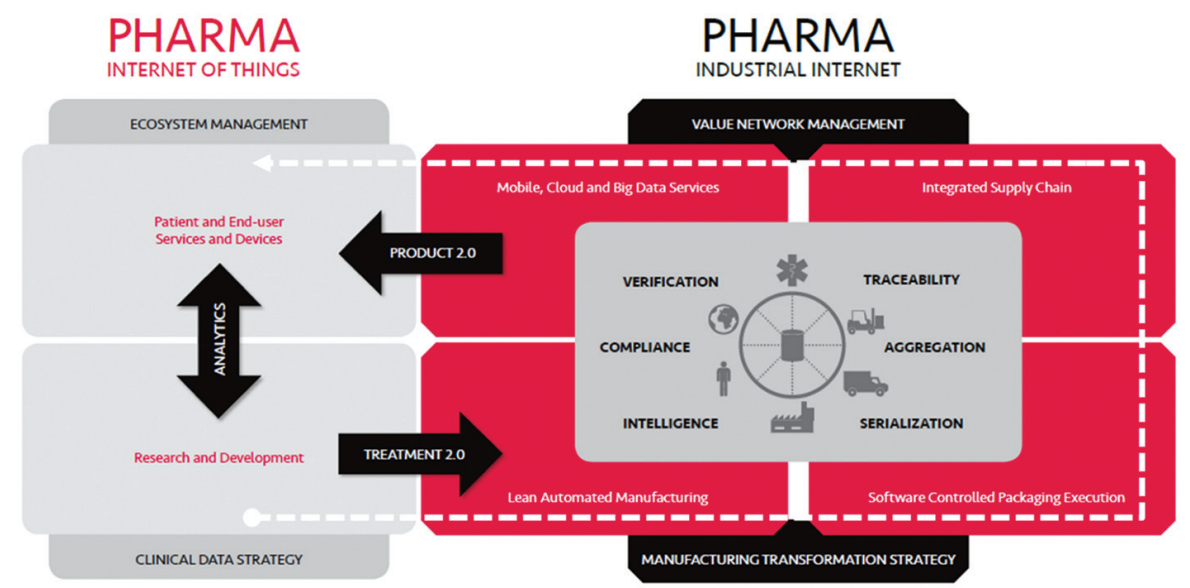

Figure 7 Pharma industrial internet ecosystem from the product lifecycle and supply network view and the relation with the pharma internet of things services. the white dotted line represents the product lifecycle from medicine R\&D to the actual medicinall product usage.

Pharma Industrial Internet ecosystem consists of four different yet complementing pharma service infrastructures (Figure 7). The foundation for the infrastructure implementations is the compliance with the regulatory, customer and internal operational requirements. Simply put, without the validated manufacturing and product supply compliances there's no business. This makes implementing the industrial internet drastically more complex and expensive compared to any other industry.

\subsection{Lean Automated Manufacturing}

Manufacturing consists of operations responsible for the primary manufacturing i.e. producing the medicine or treatment. The key platforms supporting Industrial Internet technologies are SCADA (Supervisory Control And Data Acquisition), Distributed Production Control (DCS) and Manufacturing Execution Services (MES). These technologies are already in place today but are not yet fully utilized for creating industrial internet services ("intelligence") such as real-time manufacturing analytics and predictive maintenance.

Current pharma manufacturing will face substantial changes from rigid processes and proprietary infrastructures towards lean automated manufacturing. The lean manufacturing operations enable manufactures to better adapt to the changing customer and market needs: both with solutions and processes. This brings agility and flexibility in the manufacturing but at the same time 
also improves understanding and management of the manufacturing processes. This is enabled with intelligence both in terms of intelligent manufacturing (higher flexibility in process automation) and manufacturing intelligence (applying better process knowledge to improve the production).

\subsection{Software Controlled Packaging Execution}

Packaging operations, or finished manufacturing, is responsible for the getting the medicine into a sellable and distributable form i.e. sales packages. In addition to the manufacturing technologies, the key industrial internet enabler is Packaging Execution Service (PES) enabling the transition from manual packaging into full automated packaging with predictive maintenance capabilities.

The first pharma service infrastructure to be adapted with Pharma Industrial Internet services is the packaging in the finished medicines manufacturing. This is facilitated by the global regulatory requirements for the medicine serialization, aggregation and traceability. Serialization will require moving from manual operated packaging processes to automation enabled by the software controlled packaging execution [12]. This requires packaging execution systems which will connect different the enterprise IT systems with the manufacturing infrastructure such as analytics, product master data, serial number repositories and maintenance planning. This sounds obvious but is not mostly the case at the moment in the pharma industry: manufacturing is mostly isolated from enterprise IT, with the potential exception of ERP.

Another important enabler for the Pharma Industrial Internet services is aggregation which enables pharma companies to improve their warehousing and logistical capabilities. Aggregation is the process of building a relationship between unique identifiers assigned to packaging containers. This helps the pharma companies significantly improve warehousing and logistical transparency leading to better demand predictability and hence, improved profitability.

\subsection{Integrated Supply Chain}

Integrated supply chain consists of complex network of storage and logistics and takes care of getting the sellable products into the market, and ultimately to patients. Here the key industrial internet technologies are mobile technologies, smart warehousing services and robots automating warehousing processes and inventory management, and traceability services for the supply chain transparency. 
The obvious benefit with the Pharma Industrial Internet services is the improved supply chain management. This is facilitated by the real-time traceability data and connectivity with the supply chain partners. The integrated supply chain will utilize collaborative service platforms for distributing, sharing and managing the product and logistical information. This reduces the time-to-market, improves the supply transparency to the customers and decreases the wastage.

\subsection{Mobile, Cloud and Big Data Enterprise IT Services}

Enterprise IT services are supporting all the other infrastructures in offering the general IT services such as ERP and archiving services, but also have their own distict industrial internet services such as analytics and external manufacturing partner collaboration services. A new service enabled by the new industrial internet service infrastructure is the medicine verification. This will be required to ensure that the genuine medicine is being supplied to the right market at the right time and for the right patient.

The existing enterprise IT services such as ERP, CRM, supply chain management, sales management and even marketing platforms can benefit of getting more precise and real-time data from the supply network. In addition to the existing enterprise IT services, pharma companies are increasingly already investing to new service capabilities such as analytics and mobile patient services, or investigating moving some of the existing IT services to cloud such as product supply traceability and partner collaboration services.

In addition to the service infrastructures, Pharma Industrial Internet enables changes in business models and actual manufacturing operations. For example, using industrial internet solutions pharma manufacturers are able to manage more complex value creation in terms of manufacturing partner collaboration, and product distribution. With the high automation and real-time global connectivity, the medicine manufacturing and packaging can become commodity and distributed globally. The third-party production can be then managed centrally by the pharma company, and yet the product can be traced and verified on a sales unit level throughout the supply network up to the point of dispense.

\section{Implementing Pharma Industrial Internet}

Although the transition from traditional legacy pharma manufacturing and IT solutions towards implementing Pharma Industrial Internet solutions has slowly started, it will no doubt take at least a decade before we see the majority 
of the pharma companies adopting these solutions. The first pharma business operations to adopt the industrial internet will be finished medicines manufacturing (packaging) due to the global regulatory compliance requirements for the medicine verification and traceability [12]. At the same time, other business operations (primary manufacturing, integrated supply chain and enterprise IT services) are gradually adopting the next generation solutions mainly for the improved regulatory compliance, competitiveness and cost-efficiency.

There can be seen a clear linkage between the new pharma reality to key technology enablers and industrial internet architectures (Table 1). The actual implementations will be naturally combining different technologies, service platforms and solutions from various different vendors. Some of the solutions will be deployed on-premise as currently but increasingly the solutions will be provisioned as cloud services for example for the cost-efficiency and scalability reasons.

$5 \mathrm{G}$ service platforms can play substantial role when deploying cloud based industrial internet services using pharma standards. The true risk is whether the $5 \mathrm{G}$ service infrastructure standardization will be ever specified up to the IaaS level to create the foundation for IIRA based open industrial internet infrastructures (Figure 4). The competing approach will be deploying the industrial internet solutions on top of proprietary IaaS service infrastructures from global IaaS platform vendors like Amazon, Google, Microsoft, Nokia and IBM. This can mean that the biggest proponents for $5 \mathrm{G}$, telecom operators, would remain as (fast) bit pipe providers for the industrial internet services. Interestingly, the global IaaS platform vendors are already collaborating on an open IaaS initiative called Telecom Infra Project which may help telecom operators to maintain the competitiveness for implementing the IoT and industrial internet service infrastructures on top of 5G networking infrastructure [15].

\section{Conclusions}

Pharma product supply is facing the biggest disruption since the introduction of manufacturing automation in 1980s. The disruption, called 'the new pharma reality' is driven by the increasing global regulation, intensifying competition, more demanding customer requirements and increasingly complicated global ecosystems.

The new pharma reality will pave the way for the industrial internet implementations in pharma manufacturing, packaging, supply chain and service industries with 
158 P. Kemppainen

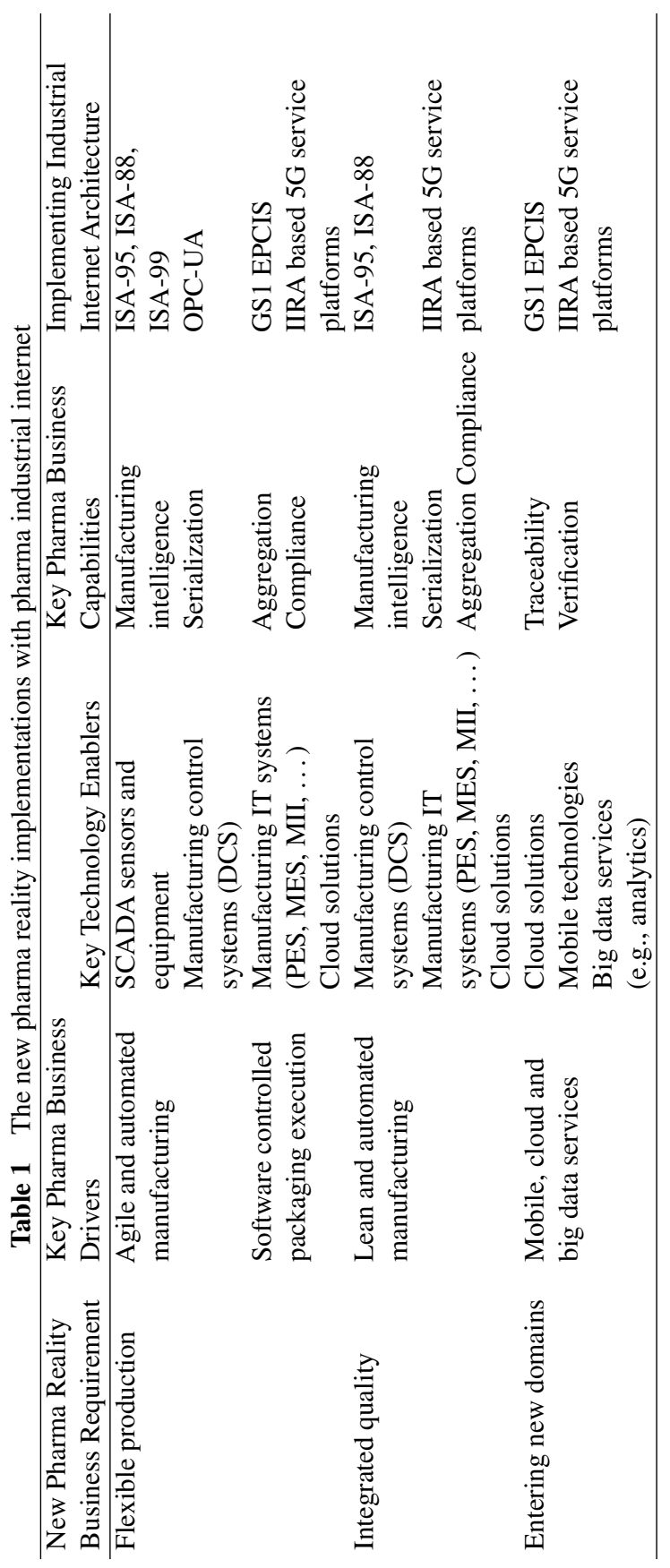


- Agile and automated manufacturing with intelligent machinery and equipment.

- Fully automated, software controlled manufacturing and packaging execution.

- Cloud based medicine data repositories for regulatory compliance and ePedigree services.

- Big data and cloud services for manufacturing, inventory, supply chain optimization and customer/patient engagement.

- Complex value network with connected suppliers, customers and service providers.

The Pharma Industrial Internet reference model uses three different architectures for specification and modelling:

- 5G Public Private Partnership (5G PPP) for the industrial internet ecosystem, network access and service delivery infrastructure,

- Industrial Internet Consortium Reference Architecture (IIRA) for modelling the industrial internet services functionality and distribution, and

- Standards and specification used at the pharma industry to specify the functional requirements and processes for the implementations.

The primary goal with the Pharma Industrial Internet is to provide a holistic view on the pharma digitalization transformation in the pharma manufacturing and supply network. It also helps pharma companies to conceptualize and implement pharma regulatory compliant industrial internet solutions using global industry standards and proven architecture frameworks fulfilling the future IT/OT infrastructure, business operations and expenditure requirements for the new pharma reality and beyond.

\section{References}

[1] Moelgaard, G. (2015). The Biggest Challenges in a New Pharma Reality, NNE Pharmaplan. Available at: http://www.nnepharmaplan.com/insights/ on-the-move/the-biggest-challenges-in-a-new-pharma-reality/[accessed May 12, 2016].

[2] Pharmaplan, N. N. E. (2015). Angle. February 2015 (in print).

[3] World Economic Forum (2015). Industrial Internet of Things: Unleashing the Potential of Connected Products and Services. Available at: http:// www3.weforum.org/docs/WEFUSA_IndustrialInternet_Report2015.pdf [accessed March 20, 2016]. 
[4] Accenture Technology Review (2015). Driving Unconventional Growth Through the Industrial Internet of Things. Available at: https:// www.accenture.com/us-en/_acnmedia/Accenture/next-gen/reassembling -industry/pdf/Accenture-Driving-Unconventional-Growth-through-IIoT. pdf [accessed March 20, 2016].

[5] 5GPPP (2015). 5G Vision. Available at: https://5g-ppp.eu/wp-con tent/uploads/2015/02/5G-Vision-Brochure-v1.pdf [accessed March 12, 2016].

[6] Industrial internet consortium (2016). Industrial Internet Reference Architecture. Available at: http://www.iiconsortium.org/IIRA.htm [accessed March 12, 2016].

[7] International Society for Automation (2016). ISA95 Enterprise-Control System Integration. Available at: https://www.isa.org/isa95/ [accessed March 12, 2016].

[8] International Society for Automation (2016). ISA88 Batch Control. Available at: https://www.isa.org/isa88/ [accessed March 12, 2016].

[9] International Society for Automation (2016). ISA99 Industrial Automation and Control Systems Security. Available at: https://www.isa.org/ isa99/ [accessed March 12, 2016].

[10] GS1 (2014). The Global Language of Business: EPCIS and Core Business Vocabulary. Available at: http://www.gs1.org/epcis [accessed March 12, 2016].

[11] OPC Foundation (2008). Unified Architecture. Available at: https://opc foundation.org/about/opc-technologies/opc-ua/ [accessed March 12, 2016].

[12] Pharmaplan, N. N. E. (2015). Get on the Right Track, Serialisation. April 2015 (in print).

[13] Industrial internet consortium (2015). Deliverable. Available at: http://www.iiconsortium.org/IIC_Deliverables-May-2015.pdf [accessed March 12, 2016].

[14] Industrial internet consortium (2015). Industrial Internet Reference Architecture. Available at: http://www.iiconsortium.org/IIRA-1-7ajs.pdf [accessed March 12, 2016].

[15] Tervonen, H. (2016). Reinvigorating the Telecommunications Landscape. Available at: https://telecominfraproject.com/news/nokia-reinvigo rating-the-telecommunications-landscape [accessed March 20, 2016]. 


\section{Biography}

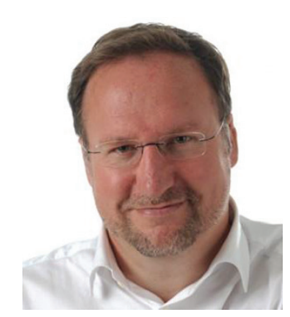

Mr. P. Kemppainen is entrepreneur and executive consultant in strategic digitalization advisory and initiatives for biopharma and pharma companies. The customer assignments range from helping with the clinical trials and patient care digitalization (IoT) strategies to implementing the global regulatory compliances in medicine serialization and traceability (Industrial Internet). In this capacity, he is also a senior consultant and advisor for NNE Pharmaplan, a leading life science engineering and consulting company.

Mr. Kemppainen was Chief Executive Officer at Original1 GmbH (a joint venture of SAP, Nokia and Giesecke\&Devrient - leaders in enterprise IT, mobile and security) servicing a cloud and mobile platform for product serialization, traceability and consumer authentication services in Europe and China. In Europe, Originall focused in serialization and traceability services for pharma, consumer products and manufacturing industries. In China, Original 1 commercialized the first drug traceability and authentication cloud and mobile service for pharma companies and consumers utilizing China FDA Electronic Drug Monitoring Service.

Prior to Original1 he headed Nokia Authentication Solution business in Nokia China empowering consumers and businesses to authenticate product originality and counterfeits using their mobile phones. The products covered consumer products, drugs and ID cards and ultimately the solution was selected as the best anti-counterfeit solution in China by China RFID Journal and China Anti-counterfeit Association. Before heading Nokia Authentication Solution business, he led the incubation of new businesses at Nokia Ventures Organization.

Currently Mr. Kemppainen is also working on Ph.D. dissertation on pharma digitalization technology and business disruption (IoT/Industrial Internet) at Lappeenranta University of Technology Faculty of Industrial Engineering and Management. 
